\title{
BMJ Antiviral therapy for prevention of Open hepatocellular carcinoma and mortality in chronic hepatitis B: systematic review and meta-analysis
}

\author{
Maja Thiele, ${ }^{1}$ Lise L Gluud, ${ }^{2}$ Emilie K Dahl, ${ }^{3}$ Aleksander Krag ${ }^{1}$
}

To cite: Thiele M, Gluud LL, Dahl EK, et al. Antiviral therapy for prevention of hepatocellular carcinoma and mortality in chronic hepatitis B: systematic review and meta-analysis. BMJ Open 2013;3:e003265.

doi:10.1136/bmjopen-2013003265

- Prepublication history and additional material for this paper is available online. To view these files please visit the journal online (http://dx.doi.org/10.1136/ bmjopen-2013-003265)

Received 21 May 2013 Revised 8 July 2013 Accepted 10 July 2013
For numbered affiliations see end of article.

\section{Correspondence to} Dr Maja Thiele; maja.thiele@rsyd.dk

\section{ABSTRACT}

Objectives: The effect of antiviral therapy on clinical outcomes in chronic hepatitis B virus (HBV) is not established. We aimed to assess the effects of interferon and/or nucleos(t)ide analogues versus placebo or no intervention on prevention of hepatocellular carcinoma (HCC) and mortality in chronic HBV.

Design: Random-effects pairwise meta-analysis of randomised trials and observational studies.

Setting: Electronic and manual searches were combined. Randomised controlled trials (RCTs) were included in the primary analyses. Observational studies were included in sensitivity analyses.

Primary and secondary outcome measures: The primary outcome measures were $\mathrm{HCC}$ incidence and mortality. The secondary outcome measure was HCC mortality.

Results: We included 8 RCTs, 8 prospective cohort studies and 19 case-control studies with a total of 3433 patients allocated to antiviral therapy and 4625 controls. The maximum duration of follow-up was 23 years. Randomised trials found no effect of antiviral therapy on HCC or mortality. Cohort studies found that antiviral therapy increased the risk of $\mathrm{HCC}$ (risk ratio 1.43; $95 \% \mathrm{Cl}$ 1.06 to 1.95), whereas case-control studies found a decreased risk of HCC in the intervention group (risk ratio $0.69 ; 95 \% \mathrm{Cl} 0.54$ to 0.88 ). There was a clear difference between the results of RCTs and observational studies (test for subgroup differences, $p<0.001$ ). Antiviral therapy did not affect mortality in cohort studies, but reduced mortality in case-control studies (relative risk $0.71 ; 95 \%$ $\mathrm{Cl} 0.54$ to 0.93 ; test for subgroup differences, $\mathrm{p}=0.406$ ). Conclusions: The effect of antiviral therapy on clinical outcomes in HBV remains to be established. Although there was a positive effect in the sensitivity analyses, the strength of the evidence does not allow for extrapolation to clinical practice as research design plays an essential role in the overall assessment.

Trial registration number: Prospero number CRD42013003881.

\section{INTRODUCTION}

Worldwide, two billion people have been infected with hepatitis B virus (HBV).

\section{ARTICLE SUMMARY}

Article focus

- The effect of antiviral treatment for chronic hepatitis $B$ has been assessed using surrogate markers.

- An evaluation of the effect on hepatocellular carcinoma and mortality is missing.

Key messages

- Research design plays an essential role on the hepatocellular carcinoma incidence estimates. As prospective cohorts and case-control series show opposing results, the reports from such trials should be interpreted with caution.

- Sensitivity analyses show a positive effect of treatment on mortality.

Strengths and limitations of this study

- A large number of observational studies were included that allowed for detailed sensitivity analyses with tests for subgroup differences.

- Only eight randomised controlled trials were included.

- The effect of modern nucleos(t)ides could not be assessed as newer trials do not include placebotreated or untreated patients in the control groups.

Chronic HBV may lead to hepatocellular carcinoma (HCC), cirrhosis and liver failure, and each year about 600000 people die due to hepatitis. ${ }^{1-3}$ Globally, HCC is the fifth most common cause of cancer deaths in men, and the sixth in women. ${ }^{4-6}$ Vaccine programmes have decreased the incidence of $\mathrm{HBV},{ }^{7}$ but mortality from HBV-related HCC and cirrhosis is increasing due to the high prevalence of chronically infected patients. ${ }^{9} 10$ The aim of antiviral treatment is to prevent progression to these clinical outcome measures. ${ }^{11-13}$ Recommended treatments include interferon and nucleos $(\mathrm{t})$ ide analogues (NA). ${ }^{14}{ }^{15}$ A viral response may reduce the risk of $\mathrm{HCC},{ }^{12}$ but the results of clinical studies and meta-analyses on antiviral 
therapy are not consistent. ${ }^{16-24}$ One meta-analysis ${ }^{25}$ found that antiviral therapy decreased liver-related mortality, whereas a cohort series found decreased overall mortality in patients with a viral response to interferon. ${ }^{26}$ On the other hand, randomised controlled trials (RCTs) have failed to show an effect on HCC or mortality. ${ }^{27}{ }^{28}$ We therefore conducted a systematic review of the evidence on antiviral treatment for prevention of HCC and mortality in patients with HBV.

\section{METHODS}

Scope

This systematic review evaluates the effects of antiviral therapy versus placebo or no intervention on prevention of HCC and mortality in patients with HBV. The review is based on a registered written protocol (Prospero number CRD42013003881) according to the methods specified in the Cochrane Handbook for Reviews on Interventions $^{29}$ and the MOOSE Guidelines for Meta-Analyses and Systematic Reviews of Observational Studies. ${ }^{30}$ For a more detailed description of the methods, please see the MOOSE checklist.

\section{Data sources}

Eligible trials were identified through electronic and manual searches. Electronic searches were performed in MEDLINE (1966-2012), EMBASE (1928-2012) and Web of Science (1900-2012). Literature searches included keywords for HCC, chronic HBV and antiviral treatment. Manual searches included scanning of reference lists in relevant papers and conference proceedings and the International Clinical Trials Registry Platform.

\section{Study selection}

Our primary analyses included RCTs (primary analyses) on antiviral interventions (interferon and/or NA) versus placebo or no intervention for patients with HBV who had not previously received antiviral therapy (treatment naïve). Owing to the expected prognosis and the duration of follow-up necessary to evaluate intervention effects on clinical outcome measures in HBV, observational studies were included in sensitivity analyses. The primary outcome measures were HCC diagnosed using recommended criteria $^{31}{ }^{32}$ and all-cause mortality. To avoid prevalent cases of HCC, the outcomes were assessed after at least 12 months of follow-up. Some studies did not perform screening ultrasonography and would therefore not detect small HCC present at inclusion. Hence, 12 months was chosen as a limit. The secondary outcome measure was HCC-related mortality.

\section{Data extraction and quality assessment}

Two authors extracted data independently. When data were not available in the published reports, additional information was retrieved through correspondence with the primary investigators.
The Cochrane Collaboration's Tool for Assessing Risk of Bias was used to evaluate bias control in RCTs. The assessment included the randomisation methods (allocation sequence generation and allocation concealment), blinding (of participants, personnel and investigators), completeness of outcome data, reporting of data and other biases. ${ }^{33}$ All observational studies were classed as having a high risk of bias. Based on the MOOSE guidelines, the assessment of potential sources of bias within observational studies included documentation of how data were classified and coded (multiple raters, blinding and inter-rater reliability), assessment of confounding (comparability of cases and controls in studies where appropriate) and blinding of quality assessors, stratification or regression on possible predictors of study results.

\section{Data synthesis and analysis}

Statistics were performed using Stata V.12 (Statacorp, College Station, Texas, USA) and Trial Sequential Analysis (CTU, Copenhagen, Denmark). Meta-analyses were performed with results expressed as risk ratios, 95\% CI and $\mathrm{I}^{2}$ as a marker of heterogeneity. For meta-analyses showing a statistically significant effect, the number needed to treat was calculated based on the risk difference. Initial sensitivity analyses included repeating all meta-analyses using both random and fixed effect models. The results of these analyses were only reported if the conclusions differed. Regression analyses were performed to assess for publication bias and other smallstudy effects (Egger's test). Sequential analyses were performed for meta-analyses showing an intervention effect after adjusting for the risk of bias associated with cumulative testing. ${ }^{34}$ The sequential analysis was performed using a random effects model, $\alpha(5 \%)$, power $(80 \%)$ and the incidence rates and the intervention effects identified in the meta-analyses. Preplanned sensitivity analyses were performed with the inclusion of observational studies. These analyses were performed stratified by study design (RCT, prospective cohort or casecontrol study) and with fixed-effect inverse variance models that compared the results of subgroups. The result of the subgroup comparisons was expressed as $p$ values (test for subgroup differences). Additional sensitivity analyses were performed to evaluate the influence of bias control (limiting the analysis to trials with adequate randomisation), the type of antiviral therapy (comparing interferon, NA or both) and the effect of HCC screening (comparing the results of trials with or without screening). Finally, subgroup analyses including only patients with cirrhosis were performed.

\section{RESULTS}

\section{Literature searches and study inclusion}

The electronic and manual searches identified 27474 potentially relevant records (figure 1). After excluding duplicates and studies that did not fulfil our inclusion 


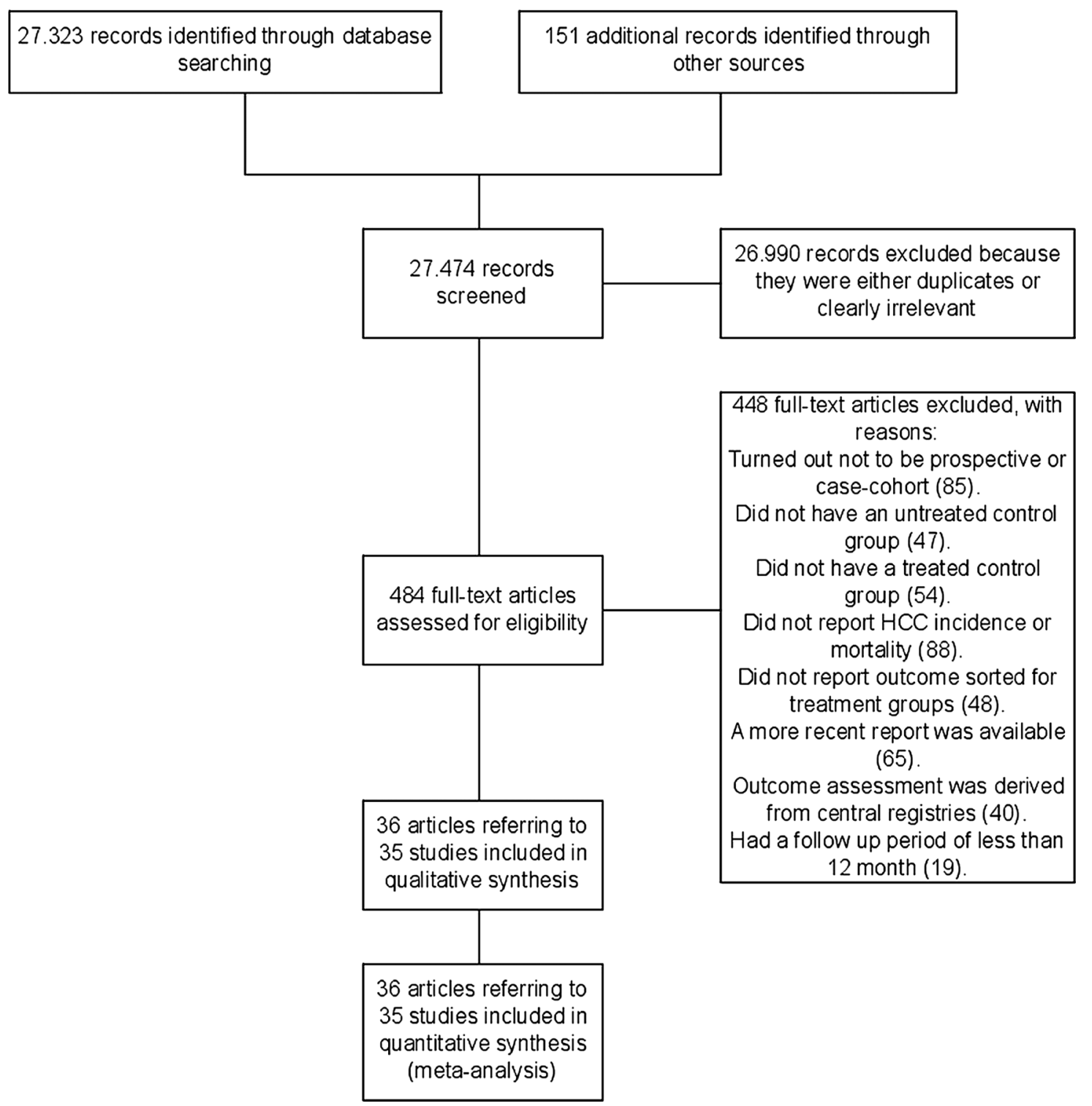

Figure 1 Flow diagram of the study.

criteria, 36 references referring to 8 RCTs, 8 prospective cohort studies and 19 case-control studies were included. ${ }^{26-28} 35-67$

\section{Characteristics of the included RCTs and observational studies}

The RCTs were conducted in Europe $(n=4)$, Asia $(n=2)$ and Africa $(n=2)$. The duration of follow-up ranged from 1 to 11 years. One trial performed HCC screening. Six trials assessed interferon and two trials focused on NA (table 1). A total of 840 patients received antiviral therapy and 447 patients received placebo or no intervention. The proportion of men ranged from $70 \%$ to $100 \%$ and the mean age ranged from 33 to 44 years. The proportion of patients with cirrhosis at inclusion ranged from $0 \%$ to $66 \%$ (table 2). The proportion of patients with a virological response ranged from $7 \%$ to $58 \%$ in the treatment group and from $1 \%$ to $22 \%$ in controls. A biochemical response was achieved in $14-66 \%$ of patients in the treatment group and in $1-20 \%$ of controls. The randomisation methods were described as adequate in three trials (table 3).

The prospective cohorts and case-control studies were conducted in Europe $(n=12)$, Asia $(n=13)$, North America $(n=1)$ and South America $(n=1)$. The duration of follow-up ranged from 2 to 23 years. HCC screening was performed in all prospective cohort studies and in 13 of the case-control studies. In total, 18 studies assessed interferon, 7 assessed NA and 2 combined therapy with interferon and NA (table 1). A total of 2593 patients received antiviral therapy and 4178 patients received no intervention. The proportion of men ranged from $53 \%$ to $95 \%$ and the mean age ranged from 27 to 65 years. The proportion of patients with cirrhosis ranged from $0 \%$ to $100 \%$ (table 2). In the prospective cohorts, the proportion of patients with a virological response in the treatment and control groups was $23-69 \%$ and $0-23 \%$, respectively. A biochemical 
Table 1 Characteristics of trials

\begin{tabular}{|c|c|c|c|c|c|c|}
\hline Study, year (reference) & $\begin{array}{l}\text { Country of } \\
\text { origin }\end{array}$ & Intervention (dose) & $\begin{array}{l}\text { Number of } \\
\text { patients }\end{array}$ & $\begin{array}{l}\text { Follow-up (mean/ } \\
\text { median year) }\end{array}$ & $\begin{array}{l}\text { HCC screening } \\
\text { (yes/no) }\end{array}$ & Outcomes reported \\
\hline \multicolumn{7}{|l|}{ Randomised controlled trials } \\
\hline Anderson et al, $1987^{35}$ & England & IFN $\left(2.5-7.5 \mathrm{MU} / \mathrm{m}^{2} / \mathrm{d}\right)$ & $\begin{array}{l}\text { I } 14 \\
\text { C } 16\end{array}$ & $\begin{array}{l}\text { I } 1.0 \\
\text { C } 1.0\end{array}$ & No & Overall mortality \\
\hline Chan et al, $2007^{39}$ & China & Lamivudine (100 mg/d) & $\begin{array}{l}\text { I } 89 \\
\text { C } 47\end{array}$ & $\begin{array}{l}\text { I } 2.5 \\
\text { C } 2.5\end{array}$ & No & HCC incidence \\
\hline Farci et al, $2004^{43}$ & Italy & IFN (3-9 MU/×3w) & $\begin{array}{l}\text { I } 28 \\
\text { C } 10\end{array}$ & $\begin{array}{l}\text { I } 10.8 \\
\text { C } 10.8\end{array}$ & No & Overall mortality \\
\hline Krogsgaard, $1998^{28}$ & Europe & IFN $(1.5-18 \mathrm{MU} / \times 3 \mathrm{w})$ & $\begin{array}{l}\text { I } 210 \\
\text { C } 98\end{array}$ & $\begin{array}{l}\text { I } 1.3 \\
\text { C } 1.3\end{array}$ & No & $\begin{array}{l}\text { HCC incidence and } \\
\text { mortality }\end{array}$ \\
\hline Liaw et al, $2004^{27}$ & Asia & Lamivudine (100 mg/d) & $\begin{array}{l}\text { I } 436 \\
\text { C } 215\end{array}$ & $\begin{array}{l}\text { I } 2.7 \\
\text { C } 2.7\end{array}$ & Yes & $\begin{array}{l}\text { HCC incidence } \\
\text { Overall mortality }\end{array}$ \\
\hline Mazzella et al, $1999^{53}$ & Italy & IFN (648 MU total) & $\begin{array}{l}\text { I } 33 \\
\text { C } 31\end{array}$ & $\begin{array}{l}17.2 \\
\text { C } 6.6\end{array}$ & No & $\begin{array}{l}\text { HCC incidence and } \\
\text { mortality }\end{array}$ \\
\hline Robson et al, $1992^{56}$ & South Africa & IFN (10 MU/×3w) & $\begin{array}{l}\text { I } 10 \\
\text { C } 10\end{array}$ & $\begin{array}{l}\text { I } 1.4 \\
\text { C } 1.4\end{array}$ & No & Overall and HCC mortality \\
\hline Waked et al, $1990^{62}$ & Egypt & $\begin{array}{l}\text { IFN }\left(5 \mathrm{MU} / \mathrm{m}^{2} / \times 3 \mathrm{w}-\right. \\
\left.5 \mathrm{MU} / \mathrm{m}^{2} / \mathrm{d}\right)\end{array}$ & $\begin{array}{l}\text { I } 20 \\
\text { C } 20\end{array}$ & $\begin{array}{l}\text { I } 1.3 \\
\text { C } 1.3\end{array}$ & No & Overall and HCC mortality \\
\hline \multicolumn{7}{|l|}{ Prospective cohorts } \\
\hline Benvegnu et al, $1998^{36}$ & Italy & IFN (5-10 MU/×3w) & $\begin{array}{l}\text { I } 13 \\
\text { C } 24\end{array}$ & $\begin{array}{l}\text { I } 6.0 \\
\text { C } 6.0\end{array}$ & Yes & $\begin{array}{l}\text { HCC incidence } \\
\text { Overall mortality }\end{array}$ \\
\hline Brunetto et al, $2002^{38}$ & Italy & IFN (9 MU/×3w) & $\begin{array}{l}\text { I } 103 \\
\text { C } 61\end{array}$ & $\begin{array}{l}16.0 \\
\text { C } 6.0\end{array}$ & Yes & Overall mortality \\
\hline $\begin{array}{l}\text { Chan et al, } 2012^{40} \\
\text { Wong et al, } 2010^{63}\end{array}$ & China & $\begin{array}{l}\text { Nucleos(t)ides IFN } \\
\text { (NS) }\end{array}$ & $\begin{array}{l}\text { I } 158 \\
\text { C } 1271\end{array}$ & $\begin{array}{l}\text { I } 10.1 \\
\text { C } 10.1\end{array}$ & Yes & $\begin{array}{l}\text { HCC incidence, overall and } \\
\text { HCC mortality }\end{array}$ \\
\hline Di Marco et al, $1999^{42}$ & Italy & IFN (NS) & $\begin{array}{l}\text { I } 109 \\
\text { C } 193\end{array}$ & $\begin{array}{l}17.8 \\
\text { C } 7.8\end{array}$ & Yes & Overall mortality \\
\hline Ma et al, $2008^{49}$ & China & Nucleos(t)ides (NS) & $\begin{array}{l}\text { I } 41 \\
\text { C } 176\end{array}$ & $\begin{array}{l}\text { I } 2.9 \\
\text { C } 2.9\end{array}$ & Yes & Overall mortality \\
\hline Mazzella et al, $1996^{52}$ & Italy & IFN (10 MU/×3w) & $\begin{array}{l}\text { I } 34 \\
\text { C } 28\end{array}$ & $\begin{array}{l}\text { I } 4.1 \\
\text { C } 4.0\end{array}$ & Yes & HCC incidence \\
\hline Papatheodoridis et al, $2001^{55}$ & Greece & IFN (3 MU/×3w) & $\begin{array}{l}\text { I } 209 \\
\text { C } 152\end{array}$ & $\begin{array}{l}16.0 \\
\text { C } 6.1\end{array}$ & Yes & $\begin{array}{l}\text { HCC incidence, overall and } \\
\text { HCC mortality }\end{array}$ \\
\hline Tong et al, $2006^{59}$ & USA & IFN (NS) & $\begin{array}{l}\text { I } 22 \\
\text { C } 378\end{array}$ & $\begin{array}{l}17.0 \\
C 7.0\end{array}$ & Yes & $\begin{array}{l}\text { HCC incidence, overall and } \\
\text { HCC mortality }\end{array}$ \\
\hline \multicolumn{7}{|l|}{ Case-control series } \\
\hline Bolukbas et al, $2006^{37}$ & Turkey & Lamivudine (100 mg/d) & $\begin{array}{l}\text { I } 23 \\
\text { C } 15\end{array}$ & $\begin{array}{l}\text { I } 1.5 \\
\text { C } 2.0\end{array}$ & Yes & Overall and HCC mortality \\
\hline Das et al, $2010^{41}$ & India & $\begin{array}{l}\text { Lamivudine Adefovir } \\
\text { (NS) }\end{array}$ & $\begin{array}{l}\text { I } 151 \\
\text { C } 102\end{array}$ & $\begin{array}{l}\text { I } 4.0 \\
\text { C } 3.8\end{array}$ & Yes & $\begin{array}{l}\text { HCC incidence, overall and } \\
\text { HCC mortality }\end{array}$ \\
\hline Fattovich et al, $1997^{44}$ & Europe & $\begin{array}{l}\text { IFN ( } 36 \mathrm{MU} \text { to } \\
>300 \mathrm{MU} \text { total) }\end{array}$ & $\begin{array}{l}140 \\
\text { C } 50\end{array}$ & $\begin{array}{l}16.2 \\
\text { C } 6.2\end{array}$ & No & $\begin{array}{l}\text { HCC incidence, overall and } \\
\text { HCC mortality }\end{array}$ \\
\hline
\end{tabular}




\begin{tabular}{|c|c|c|c|c|c|c|}
\hline Study, year (reference) & $\begin{array}{l}\text { Country of } \\
\text { origin }\end{array}$ & Intervention (dose) & $\begin{array}{l}\text { Number of } \\
\text { patients }\end{array}$ & $\begin{array}{l}\text { Follow-up (mean/ } \\
\text { median year) }\end{array}$ & $\begin{array}{l}\text { HCC screening } \\
\text { (yes/no) }\end{array}$ & Outcomes reported \\
\hline IIHCSG, $1998^{45}$ & $\begin{array}{l}\text { Italy and } \\
\text { Argentina }\end{array}$ & IFN (9 MU/w) & $\begin{array}{l}149 \\
\text { C } 97\end{array}$ & $\begin{array}{l}15.8 \\
\text { C } 6.9\end{array}$ & Yes & HCC incidence \\
\hline Ikeda et al, $1998^{46}$ & Japan & IFN $(6 \mathrm{MU} / \times 2 \mathrm{w})$ & $\begin{array}{l}\text { I } 94 \\
\text { C } 219\end{array}$ & $\begin{array}{l}16.8 \\
C 7.0\end{array}$ & Yes & HCC incidence \\
\hline Lin et al, $2001^{47}$ & China & IFN $(5 \mathrm{MU} / \times 3 \mathrm{w})$ & $\begin{array}{l}130 \\
\text { C } 28\end{array}$ & $\begin{array}{l}12.7 \\
\text { C } 2.6\end{array}$ & No & $\begin{array}{l}\text { HCC incidence, overall and } \\
\text { HCC mortality }\end{array}$ \\
\hline Lin et al, $2007^{48}$ & China & IFN $\left(6-9 \mathrm{MU} / \mathrm{m}^{2} / \times 3 \mathrm{w}\right)$ & $\begin{array}{l}\text { I } 233 \\
\text { C } 233\end{array}$ & $\begin{array}{l}16.8 \\
\text { C } 6.1\end{array}$ & Yes & $\begin{array}{l}\text { HCC incidence and } \\
\text { mortality }\end{array}$ \\
\hline Mahmood et al, $2005^{50}$ & Japan & IFN (6 MU/d) & $\begin{array}{l}123 \\
\text { C } 68\end{array}$ & $\begin{array}{l}17.0 \\
C 7.0\end{array}$ & Yes & HCC incidence \\
\hline Manolakopoulos et al, $2004^{26}$ & Greece & Lamivudine (100 mg/d) & $\begin{array}{l}130 \\
\text { C } 30\end{array}$ & $\begin{array}{l}\text { I } 1.5 \\
\text { C } 1.8\end{array}$ & Yes & $\begin{array}{l}\text { HCC incidence, overall and } \\
\text { HCC mortality }\end{array}$ \\
\hline Matsumoto et al, $2005^{51}$ & Japan & Lamivudine (100 mg/d) & $\begin{array}{l}\text { I } 508 \\
\text { C } 231\end{array}$ & $\begin{array}{l}12.7 \\
\text { C } 5.3\end{array}$ & No & HCC incidence \\
\hline Niederau et al, $1996^{54}$ & Germany & IFN $(2-5 \mathrm{MU} / \times 3 \mathrm{w})$ & $\begin{array}{l}\text { I } 103 \\
\text { C } 53\end{array}$ & $\begin{array}{l}14.2 \\
\text { C } 3.2\end{array}$ & No & Overall mortality \\
\hline Romeo et al, $2009^{57}$ & Italy & $\begin{array}{l}\text { Lamivudine (NS) } \\
\text { IFN (6-9 MU) }\end{array}$ & $\begin{array}{l}\text { I } 102 \\
\text { C } 135\end{array}$ & $\begin{array}{l}\text { I } 22.4 \\
\text { C } 16.5\end{array}$ & Yes & $\begin{array}{l}\text { HCC incidence, overall and } \\
\text { HCC mortality }\end{array}$ \\
\hline Tangkijvanich et al, $2001^{58}$ & Thailand & IFN (3-6 MU/×3w) & $\begin{array}{l}167 \\
\text { C } 72\end{array}$ & $\begin{array}{l}\text { I } 4.9 \\
\text { C } 4.9\end{array}$ & Yes & HCC incidence \\
\hline Tong et al, $2009^{60}$ & USA & Lamivudine (NS) & $\begin{array}{l}\text { I } 27 \\
\text { C } 101\end{array}$ & $\begin{array}{l}15.3 \\
\text { C } 5.3\end{array}$ & Yes & $\begin{array}{l}\text { HCC incidence and } \\
\text { mortality }\end{array}$ \\
\hline Truong et al, $2005^{61}$ & Japan & $\begin{array}{l}\text { IFN (174-687 MU } \\
\text { total) }\end{array}$ & $\begin{array}{l}\text { I } 27 \\
\text { C } 35\end{array}$ & $\begin{array}{l}17.0 \\
\text { C } 6.2\end{array}$ & Yes & $\begin{array}{l}\mathrm{HCC} \text { incidence and } \\
\text { mortality }\end{array}$ \\
\hline Yuen et al, $2001^{64}$ & China & $\begin{array}{l}\text { IFN }\left(2.5-10 \mathrm{MU} / \mathrm{m}^{2} /\right. \\
\times 3 \mathrm{w})\end{array}$ & $\begin{array}{l}\text { I } 208 \\
\text { C } 203\end{array}$ & $\begin{array}{l}18.9 \\
C 9.0\end{array}$ & Yes & $\begin{array}{l}\text { HCC incidence and } \\
\text { mortality }\end{array}$ \\
\hline Yuen et al, $2004^{65}$ & China & IFN (NS) & $\begin{array}{l}16 \\
\text { C } 86\end{array}$ & $\begin{array}{l}\text { I } 10.5 \\
\text { C } 10.5\end{array}$ & No & HCC incidence \\
\hline Yuen et al, $2007^{66}$ & China & Lamivudine (100 mg/d) & $\begin{array}{l}\text { I } 142 \\
\text { C } 124\end{array}$ & $\begin{array}{l}17.5 \\
C 9.0\end{array}$ & Yes & HCC incidence \\
\hline Zampino et al, $2009^{67}$ & Italy & IFN $\left(5 \mathrm{MU} / \mathrm{m}^{2} / \times 3 \mathrm{w}\right)$ & $\begin{array}{l}\text { I } 41 \\
\text { C } 13\end{array}$ & $\begin{array}{l}123 \\
\text { C } 23\end{array}$ & No & HCC incidence \\
\hline
\end{tabular}


Table 2 Patient characteristics in included trials

\begin{tabular}{|c|c|c|c|c|c|c|}
\hline Study, year (reference) & $\begin{array}{l}\text { Median/mean } \\
\text { age (years) }\end{array}$ & $\begin{array}{l}\text { Proportion of } \\
\text { men (\%) }\end{array}$ & $\begin{array}{l}\text { Proportion with } \\
\text { cirrhosis (\%) }\end{array}$ & $\begin{array}{l}\text { Proportion with } \\
\text { elevated ALT (\%) }\end{array}$ & $\begin{array}{l}\text { Proportion positive } \\
\text { for } \mathrm{HBeAg}(\%)\end{array}$ & $\begin{array}{l}\text { HBeAg } \\
\text { seroconverters (n, \%) }\end{array}$ \\
\hline \multicolumn{7}{|l|}{ Randomised controlled trials } \\
\hline Anderson et al, $1987^{35}$ & $\begin{array}{l}136 \\
\text { C } 35\end{array}$ & 100 & 20 & 77 & 100 & $\begin{array}{l}\text { I } 2,14 \\
\text { C } 0,0\end{array}$ \\
\hline Chan et al, $2007^{39}$ & $\begin{array}{l}139 \\
\text { C } 39\end{array}$ & 84 & 16 & 77 & 5 & NS \\
\hline Farci et al, $2004^{43}$ & $\begin{array}{l}135 \\
\text { C } 38\end{array}$ & 83 & 66 & 100 & 2 & $\begin{array}{l}\text { I NA } \\
\text { C } 1,100\end{array}$ \\
\hline Krogsgaard, $1998^{28}$ & $\begin{array}{l}\text { I } 36 \\
\text { C } 36\end{array}$ & 81 & 19 & 100 & 100 & NS \\
\hline Liaw et al, $2004^{27}$ & $\begin{array}{l}\text { I } 43 \\
\text { C } 44\end{array}$ & 85 & 33 & 78 & 58 & NS \\
\hline Mazzella et al, $1999^{53}$ & $\begin{array}{l}\text { I } 36 \\
\text { C } 41\end{array}$ & 78 & 0 & 100 & 100 & $\begin{array}{l}\text { I } 30,91 \\
\text { C } 19,61\end{array}$ \\
\hline Robson et al, $1992^{56}$ & $\begin{array}{l}33 \\
\text { C } 31\end{array}$ & 70 & NS & 100 & 100 & $\begin{array}{l}15,50 \\
C 1,10\end{array}$ \\
\hline Waked et al, $1990^{62}$ & $\begin{array}{l}135 \\
\text { C } 35\end{array}$ & 78 & 40 & 100 & 100 & $\begin{array}{l}\text { I } 13,81 \\
\text { C } 5,33\end{array}$ \\
\hline \multicolumn{7}{|l|}{ Prospective cohorts } \\
\hline Benvegnu et al, $1998^{36}$ & $\begin{array}{l}\text { I } 57 \\
\text { C } 60\end{array}$ & 65 & 100 & NS & NS & NS \\
\hline Brunetto et al, $2002^{38}$ & $\begin{array}{l}\text { I } 40 \\
\text { C } 40\end{array}$ & 80 & 38 & NS & 0 & NA \\
\hline $\begin{array}{l}\text { Chan et al, } 2012^{40} \\
\text { Wong et al, } 2010^{63}\end{array}$ & NS & 67 & 32 & 87 & NS & NS \\
\hline Di Marco et al, $1999^{42}$ & $\begin{array}{l}\text { I } 33 \\
\text { C } 35\end{array}$ & 71 & 29 & 100 & 29 & $\begin{array}{l}\text { I } 35,32 \\
\text { C } 29,15\end{array}$ \\
\hline Ma et al, $2008^{49}$ & $\begin{array}{l}\text { I } 54 \\
\text { C } 54\end{array}$ & 72 & 100 & NS & 24 & NS \\
\hline Mazzella et al, $1996^{52}$ & $\begin{array}{l}\text { I } 48 \\
\text { C } 49\end{array}$ & 73 & 100 & NS & NS & NS \\
\hline Papatheodoridis et al, $2001^{55}$ & $\begin{array}{l}\text { I } 47 \\
\text { C } 49\end{array}$ & 83 & 31 & 100 & 0 & NA \\
\hline Tong et al, $2006^{59}$ & $\begin{array}{l}\text { I } 48 \\
\text { C } 48\end{array}$ & 71 & 35 & NS & 49 & NS \\
\hline \multicolumn{7}{|l|}{ Case-control series } \\
\hline Bolukbas et al, $2006^{37}$ & $\begin{array}{l}\text { I } 45 \\
\text { C } 46\end{array}$ & 82 & 100 & NS & 0 & NA \\
\hline Das et al, $2010^{41}$ & $\begin{array}{l}\text { I } 42 \\
\text { C } 46\end{array}$ & 91 & 100 & NS & 45 & $\begin{array}{l}\text { I } 12,13 \\
\text { C } 3,10\end{array}$ \\
\hline Fattovich et al, $1997^{44}$ & $\begin{array}{l}\text { I } 47 \\
\text { C } 45\end{array}$ & 87 & 100 & 100 & 100 & $\begin{array}{l}\text { I } 27,68 \\
\text { C } 30,60\end{array}$ \\
\hline
\end{tabular}

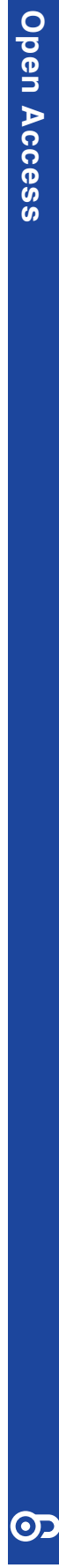




\begin{tabular}{|c|c|c|c|c|c|c|}
\hline Study, year (reference) & $\begin{array}{l}\text { Median/mean } \\
\text { age (years) }\end{array}$ & $\begin{array}{l}\text { Proportion of } \\
\text { men (\%) }\end{array}$ & $\begin{array}{l}\text { Proportion with } \\
\text { cirrhosis (\%) }\end{array}$ & $\begin{array}{l}\text { Proportion with } \\
\text { elevated ALT (\%) }\end{array}$ & $\begin{array}{l}\text { Proportion positive } \\
\text { for } \mathrm{HBeAg}(\%)\end{array}$ & $\begin{array}{l}\text { HBeAg } \\
\text { seroconverters (n, \%) }\end{array}$ \\
\hline IIHCSG, $1998^{45}$ & $\begin{array}{l}\text { I } 54 \\
\text { C } 54\end{array}$ & 64 & 100 & NS & NS & NS \\
\hline Ikeda et al, $1998^{46}$ & $\begin{array}{l}\text { I } 41 \\
\text { C } 44\end{array}$ & 79 & 100 & NS & 52 & NS \\
\hline Lin et al, $2001^{47}$ & $\begin{array}{l}\text { I } 39 \\
\text { C } 41\end{array}$ & 95 & 10 & 100 & 0 & NA \\
\hline Lin et al, $2007^{48}$ & $\begin{array}{l}132 \\
\text { C } 31\end{array}$ & 94 & 9 & NS & 100 & $\begin{array}{l}\text { I } 115,49 \\
\text { C } 86,37\end{array}$ \\
\hline Mahmood et al, $2005^{50}$ & $\begin{array}{l}\text { I } 49 \\
\text { C } 49\end{array}$ & 69 & 100 & NS & 36 & NS \\
\hline Manolakopoulos et al, $2004^{26}$ & $\begin{array}{l}165 \\
\text { C } 63\end{array}$ & 80 & 100 & 100 & 0 & NA \\
\hline Matsumoto et al, $2005^{51}$ & $\begin{array}{l}\text { I } 42 \\
\text { C } 41\end{array}$ & 73 & 18 & NS & 55 & NS \\
\hline Niederau et al, $1996^{54}$ & $\begin{array}{l}\text { I } 40 \\
\text { C } 41\end{array}$ & 78 & 28 & 100 & 100 & $\begin{array}{l}153,51 \\
\text { C } 7,13\end{array}$ \\
\hline Romeo et al, $2009^{57}$ & NS & 77 & 35 & NS & 27 & NS \\
\hline Tangkijvanich et al, $2001^{58}$ & $\begin{array}{l}\text { I } 37 \\
\text { C } 40\end{array}$ & 72 & 20 & NS & 100 & $\begin{array}{l}124,36 \\
\text { C } 7,10\end{array}$ \\
\hline Tong et al, $2009^{60}$ & $\begin{array}{l}\text { I } 46 \\
\text { C } 46\end{array}$ & 86 & 100 & 14 & 53 & NS \\
\hline Truong et al, $2005^{61}$ & $\begin{array}{l}133 \\
\text { C } 37\end{array}$ & 53 & 2 & 100 & 60 & $\begin{array}{l}19,53 \\
C 11,55\end{array}$ \\
\hline Yuen et al, $2001^{64}$ & $\begin{array}{l}127 \\
\text { C } 28\end{array}$ & 64 & NS & 32 & 100 & $\begin{array}{l}\text { I } 96,46 \\
\text { C } 93,46\end{array}$ \\
\hline Yuen et al, $2004^{65}$ & $\begin{array}{l}\text { I } 43 \\
\text { C } 43\end{array}$ & 71 & NS & NS & 23 & NS \\
\hline Yuen et al, $2007^{66}$ & $\begin{array}{l}\text { I } 34 \\
\text { C } 33\end{array}$ & 74 & 0 & 48 & 100 & NS \\
\hline Zampino et al, $2009^{67}$ & NS & 67 & 0 & NS & 54 & $\begin{array}{l}\text { I } 16,62 \\
\text { C NS }\end{array}$ \\
\hline
\end{tabular}


Table 3 Risk of bias summary

\begin{tabular}{|c|c|c|c|c|c|c|}
\hline Study, year (reference) & $\begin{array}{l}\text { Random } \\
\text { sequence } \\
\text { generation }\end{array}$ & $\begin{array}{l}\text { Allocation } \\
\text { concealment }\end{array}$ & $\begin{array}{l}\text { Blinding of } \\
\text { participants } \\
\text { and personnel }\end{array}$ & $\begin{array}{l}\text { Blinding of } \\
\text { outcome } \\
\text { assessment }\end{array}$ & $\begin{array}{l}\text { Incomplete } \\
\text { outcome } \\
\text { data }\end{array}$ & $\begin{array}{l}\text { Selective } \\
\text { reporting }\end{array}$ \\
\hline Anderson et al, $1987^{35}$ & $?$ & $?$ & + & + & $?$ & $?$ \\
\hline Chan et al, $2007^{39}$ & + & + & + & + & $?$ & $?$ \\
\hline Farci et al, $2004^{43}$ & + & + & + & + & $?$ & $?$ \\
\hline Krogsgaard, $1998^{28}$ & $?$ & $?$ & + & + & - & $?$ \\
\hline Liaw et al, $2004^{27}$ & + & + & + & + & + & + \\
\hline Mazzella et al, $1999^{53}$ & $?$ & $?$ & + & + & + & $?$ \\
\hline Robson et al, $1992^{56}$ & $?$ & $?$ & + & + & $?$ & $?$ \\
\hline Waked et al, $1990^{62}$ & $?$ & $?$ & + & + & $?$ & $?$ \\
\hline
\end{tabular}

+, Low risk of bias; -high risk of bias; ?, unknown risk of bias.

response was achieved for $23-69 \%$ of patients in the treatment groups and $31 \%$ in the control group (only reported in 1 study). In the case-control series, the proportion of patients with a virological response in the treatment and control groups ranged from 7\% to $78 \%$ and $2 \%$ to $100 \%$, respectively. A biochemical response in the two groups was $27-68 \%$ and $4-51 \%$, respectively.

\section{Prevention of HCC}

HCC was diagnosed in 22 of 768 patients in the treatment group versus 19 of 391 controls (relative risk 0.58, $95 \%$ CI 0.32 to $1.07 ; \mathrm{I}^{2}=0 \%$ ). There was no evidence of small-study effects (Egger's test, $\mathrm{p}=0.269$ ) and no difference between subgroups of trials assessing interferon or NA (test for subgroup differences, $\mathrm{p}=0.854$ ). The overall result was confirmed in sensitivity analyses including RCTs with a low risk of bias and trials with HCC screening.

Sensitivity analyses including prospective cohort studies and case-control studies were performed. In the cohort studies, HCC was diagnosed in 51 of 436 patients in the treatment group and 174 of 1853 patients in the control group. In the case-control studies, the numbers were 99 of 1778 and 201 of 1827 patients, respectively. A meta-analysis that combined RCTs and observational studies found no effect of antiviral therapy on HCC (relative risk $0.88,95 \%$ CI 0.73 to $1.05 ; \mathrm{I}^{2}=63 \%$ ). There was no evidence of small-study effects (Egger's test, $\mathrm{p}=0.730$ ). Subgroup analyses showed a clear difference between the RCTs, prospective cohorts and case-control studies (test for subgroup differences, $\mathrm{p}<0.001$; figure 2). The prospective cohort studies found that antiviral therapy increased the risk of HCC (relative risk 1.44, 95\% CI 1.06 to 1.95 ), whereas the case-control studies found that antiviral therapy reduced the risk of HCC (relative risk $0.69,95 \%$ CI 0.54 to 0.88 ). Owing to the high heterogeneity, a post hoc meta-regression analysis was performed. We evaluated the study and patient characteristics not accounted for in the sensitivity analyses, which may have influenced the result. No modifiers were found when adjusting for the following variables: proportion of men (coefficient, $-0.074 ; \mathrm{p}=0.08$ ), mean age of treated patients at inclusion (coefficient, 0.020; $\mathrm{p}=0.94$ ), mean age of untreated patients at inclusion (coefficient, 0.121; $\mathrm{p}=0.65$ ), proportion with cirrhosis at inclusion (coefficient, $-0.001 ; \mathrm{p}=0.76$ ) and region of trial (coefficient $-0.394 ; \mathrm{p}=0.55$ ).

To further evaluate the influence of bias on the overall results, we performed additional subgroup analysis in which trials were stratified for HCC screening. The analysis found 8 trials that did not perform HCC screening (relative risk $0.40,95 \%$ CI 0.26 to 0.63 ) and 18 trials that did perform HCC screening (relative risk $1.03,95 \%$ CI 0.84 to 1.25 ). The results of subgroups were clearly different (test for subgroup differences, $\mathrm{p}<0.001$ ).

Sensitivity analyses were performed to evaluate the risk of HCC among patients with cirrhosis. In the RCTs, 1 of 20 patients in the treatment group and 2 of 12 controls developed HCC (relative risk $0.75,95 \%$ CI 0.10 to 5.77 ). In the prospective cohort studies, 32 of 184 vs 142 of 482 patients developed HCC, whereas the numbers were 63 of 680 vs 161 of 955 , respectively, for case-control studies. Overall, antiviral therapy reduced the risk of HCC when including data from RCTs and observational studies (relative risk $0.74,95 \%$ CI 0.57 to $0.96, \mathrm{I}^{2}=0 \%$, number needed to treat 28 patients; figure 3). The results of RCTs and observational studies were similar (test for subgroup differences, $\mathrm{p}=0.159$ ). There was no evidence of small-study effects (Egger's test, $\mathrm{p}=0.890$ ). In the trial sequential analysis, the monitoring and $\alpha$-spending boundaries did not cross, suggesting that the result was not robust to adjustment for multiple testing.

\section{Mortality}

In the RCTs, there was no difference in mortality between the treatment and control groups (21 of 508 vs 9 of 271 patients, relative risk 1.24, $95 \%$ CI 0.58 to 2.66; $\left.\mathrm{I}^{2}=0 \%\right)$. There was no evidence of small-study effects (Egger's test, $\mathrm{p}=0.783$ ) and no difference between trials stratified by treatment (test for subgroup differences, $\mathrm{p}=0.668)$ and HCC screening $(\mathrm{p}=0.828)$. In the observational studies, the number of patients in the treatment and control groups who died was 51 of 655 vs 247 of 2231 for prospective cohort studies and 79 of 506 vs 92 


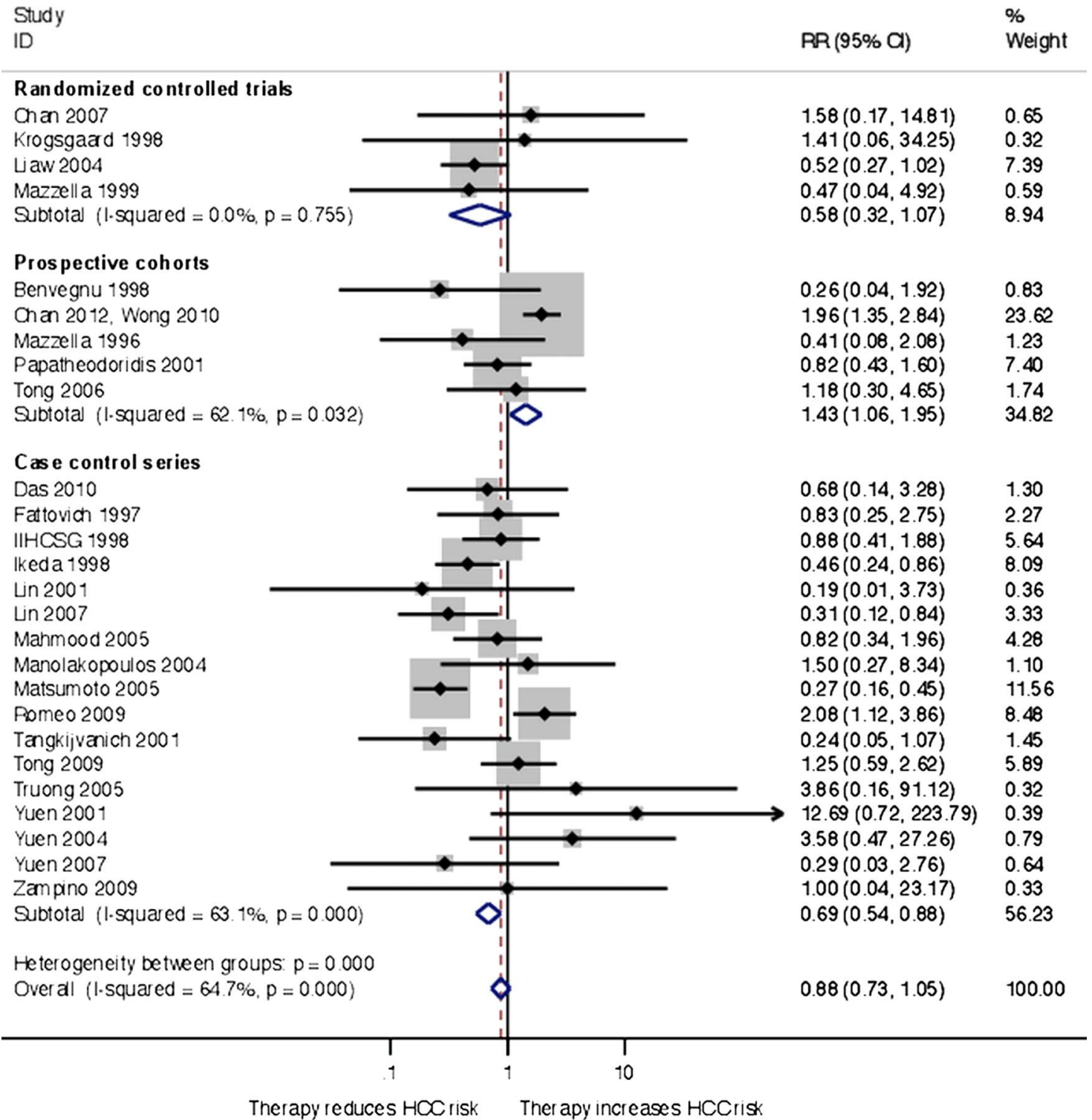

Figure 2 Random-effects inverse variance meta-analysis of antiviral therapy treatment effects on hepatocellular carcinoma in patients with chronic hepatitis $B$, subgroups according to trial design.

of 413 in the cohort studies. When combining RCTs and observational studies, random-effects meta-analysis showed that antiviral treatment decreased mortality (relative risk $0.76,95 \%$ CI 0.62 to $0.95, \mathrm{I}^{2}=14 \%$, number needed to treat 77; Egger's test, $\mathrm{p}=0.487$; figure 4). There was no difference between RCTs and observational studies (test for subgroup differences, $\mathrm{p}=0.406$ ). In the trial sequential analysis, the monitoring boundary crossed the $\alpha$-spending boundary in 2004, suggesting that the meta-analysis was robust to adjustments for multiple testing.

Only observational studies reported mortality in patients with cirrhosis. The number of patients who died in the intervention and control groups was 36 of 298 vs 141 of 499 (relative risk $0.61,95 \%$ CI 0.44 to 0.86 , $\mathrm{I}^{2}=9 \%$; number needed to treat 16 patients). There were no small-study effects (Egger's test, $\mathrm{p}=0.533$ ) and no differences between the prospective cohort and casecontrol studies (test for subgroup differences, $\mathrm{p}=0.292$ ) .

\section{HCC-related mortality}

Antiviral therapy had no effect on HCC-related mortality ( 3 of 282 vs 2 of 154 , relative risk $0.50,95 \%$ CI 0.10 to 2.44 , $\mathrm{I}^{2}=0 \% ; \mathrm{n}=2 \mathrm{RCT}$ ). Including data from observational studies had little influence on the overall result (38 of 1233 vs 144 of 2632 , relative risk $0.83,95 \%$ CI 0.5 to 1.20 , $\mathrm{I}^{2}=0 \%$; Egger's test, $\mathrm{p}=0.248$ ). There was no difference between subgroups of trials stratified by design (test for subgroup differences, $\mathrm{p}=0.481$ ).

\section{DISCUSSION}

This systematic review found that the evidence of the effect of antiviral therapy on clinical outcomes in HBV is 


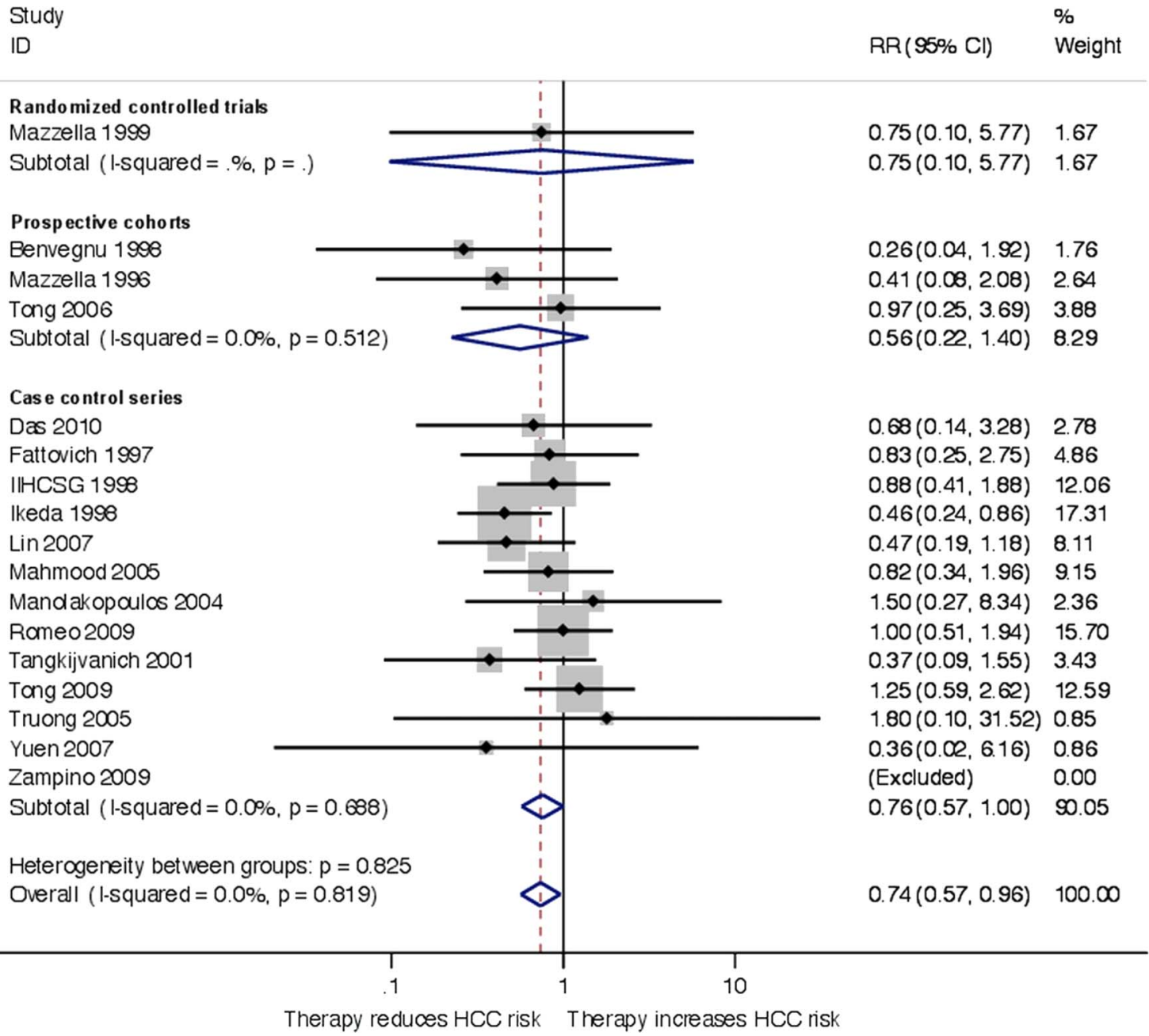

Figure 3 Random-effects inverse variance meta-analysis of antiviral therapy treatment effects on hepatocellular carcinoma in patients with chronic hepatitis B and cirrhosis, subgroups according to trial design.

weak. RCTs found no benefit of treatment on HCC, mortality or HCC-related mortality in HBV. The total number of patients and duration of follow-up may be too small to determine the clinical effects. The inclusion of observational studies did not strengthen the overall findings because there was clear evidence of bias suggesting that the study design was closely related to the estimated treatment effects. The prospective cohort studies found that antiviral therapy increased the risk of HCC and had no effect on mortality. Case-control studies found that antiviral therapy reduced HCC and mortality. These findings suggest that detection and ascertainment bias as well as confounding by indication had a considerable influence on the overall result, which may explain why previous meta-analyses have disagreed in their assessment of the benefit of antiviral therapy. ${ }^{16-18} 20212324$ The importance of detection bias was underlined in the subgroup analysis of HCC screening. No intervention effect was found in trials that performed systematic HCC screening.

The main limitation of our review is the limited number of RCTs. Only one of the included trials had prevention of
HCC as a primary outcome measure ${ }^{27}$ and none were designed to evaluate the effect on mortality or HCC-related mortality. Tests to evaluate the robustness of the results (including Egger's test) were difficult to interpret.

The current recommendation to treat patients with HBV is primarily based on surrogate outcomes. At present, the evidence supporting the use of virological markers as surrogate outcomes is weak. The fact that some studies have found a correlation between a virological response and improved liver histology does not necessarily validate their use as surrogate outcomes. Previous evidence has shown that interventions supported by surrogate markers may in fact have no benefit or even harmful effects on clinical outcome measures. ${ }^{68}$ Still, our findings are not sufficiently convincing and do not allow for changes in clinical practice.

Another limitation of the current review is our failure to extract data for analyses of treatment responders versus non-responders. However, only six cases of HCC were reportedly diagnosed in patients with biochemical or viral treatment response. This suggests that treatment response does not lead to elimination of the HCC risk, 
Study

ID
$\%$

$\mathrm{RR}(95 \% \mathrm{Cl}) \quad$ Weight

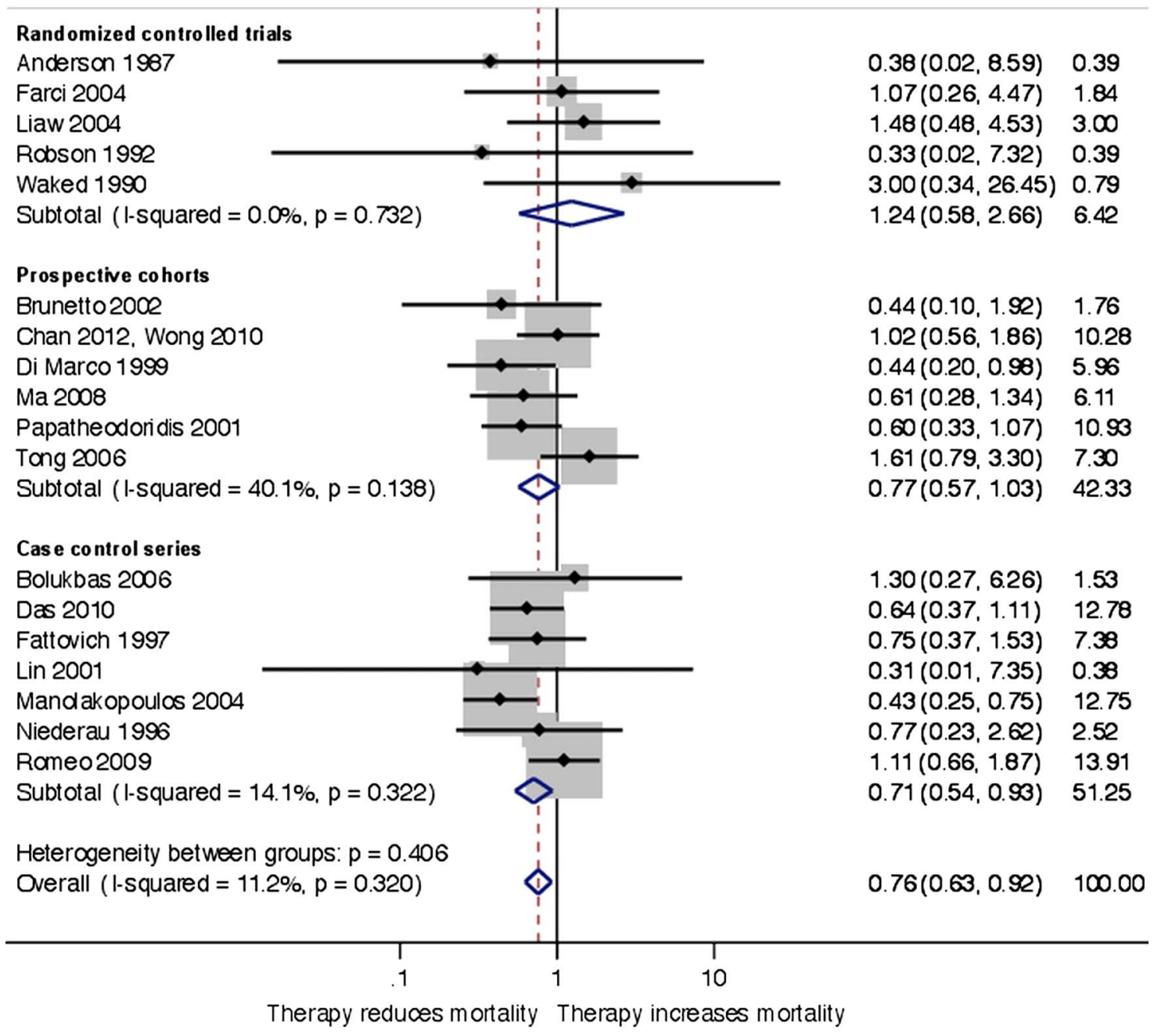

Figure 4 Random-effects inverse variance meta-analysis of antiviral therapy treatment effects on mortality in patients with chronic hepatitis $\mathrm{B}$, subgroups according to trial design.

but probably decreases HCG incidence compared to non-responders or partial responders. This would be in line with previous findings. ${ }^{19} 25$ The majority of included trials in the current review assessed first-generation NA and interferon, as reflected by the low response rates. It was, however, not within the scope of the review to investigate modern antiviral treatments, as we included untreated control groups. Newer treatments will most likely result in more patients achieving sustained suppression of HBV-DNA. It is therefore possible that the current review underestimates a potential treatment effect. It would also have been of interest had we been able to adjust for other common risk factors for HCC, such as non-alcoholic steatohepatitis, alcoholic liver disease and coinfection with hepatitis $\mathrm{C}$ virus (HCV), hepatitis D and HIV. Although data on these risk factors were extracted, there was not enough data to allow for statistical analyses.

There are several potential explanations for the discrepancies between RCTs and observational studies. ${ }^{69}$
The fact that only prospective cohort studies found an increased risk of HCC among patients receiving antiviral therapy is in opposition to speculations that the treatment affected HCC development. The findings are more likely to reflect baseline differences in the viral load, genotype and degree of liver disease. The degree of monitoring in the treatment and control groups is also likely to differ and may lead to detection bias. The importance of detection bias is further supported by the subgroup differences observed according to HCC screening. The case-control studies are likely to have an even higher risk of bias, as confounding by indication and ascertainment bias is likely to exist in retrospective studies. Reporting bias should also be considered. ${ }^{33}$

The subgroup differences with regard to the type of intervention suggest a possible anticarcinogenic effect of interferon, as seen in $\mathrm{HCV}^{70}$ We also found a decrease in HCC incidence and overall mortality in sensitivity analyses of patients with cirrhosis. This could support the case for continued treatment of patients with cirrhosis. 
We found a beneficial effect of interferon and/or NA on mortality in HBV when including RCTs and observational studies. The assessment of mortality is robust to bias. ${ }^{71}$ Accordingly, our subgroup analysis showed no clear relation between the results and the study design. HCC mortality is more prone to bias. Whether antiviral treatment for $\mathrm{HBV}$ decreases mortality except from HCC is unknown.

In conclusion, antiviral treatment for $\mathrm{HBV}$ has no proven effect on the clinical outcomes, HCC and mortality. Bias has a paramount impact on the treatment effect estimates in observational studies and we recommend a critical approach to the conclusions drawn in such studies. Future trials on antiviral treatment for HBV should be designed to show an effect on clinical endpoints rather than surrogate markers.

\section{Author affiliations}

${ }^{1}$ Department of Gastroenterology and Hepatology, Odense University Hospital, Odense, Denmark

${ }^{2}$ Department of Medicine, Copenhagen University Hospital of Gentofte,

Hellerup, Denmark

${ }^{3}$ Faculty of Health Sciences, University of Copenhagen, Copenhagen, Denmark

Contributors MT, LLG and AK conceived the idea and design, and analysed and interpreted the data. MT and EKD collected and assembled the data. MT and LLG drafted the manuscript. LLG, EKD and AK revised the manuscript for important intellectual content. All authors discussed and approved the final version of the manuscript. MT is the guarantor.

Funding This research received no specific grant from any funding agency in the public, commercial or not-for-profit sectors.

Competing interests None.

Provenance and peer review Not commissioned; externally peer reviewed.

Data sharing statement The dataset is available from the corresponding author.

Open Access This is an Open Access article distributed in accordance with the Creative Commons Attribution Non Commercial (CC BY-NC 3.0) license, which permits others to distribute, remix, adapt, build upon this work noncommercially, and license their derivative works on different terms, provided the original work is properly cited and the use is non-commercial. See: http:// creativecommons.org/licenses/by-nc/3.0/

\section{REFERENCES}

1. WHO. Position paper: Hepatitis B. WHO Weekly Epidemiological Report. World Health Organization, 2009.

2. Perz JF, Armstrong GL, Farrington LA, et al. The contributions of hepatitis $B$ virus and hepatitis $C$ virus infections to cirrhosis and primary liver cancer worldwide. J Hepatol 2006;45:529-38.

3. WHO. Hepatitis C Fact Sheet. World Health Organization, 2012. http://www.who.int/mediacentre/factsheets/fs164/en/index.html.

4. Ferlay J, Shin HR, Bray F, et al. GLOBOCAN 2008 v2.0, Cancer incidence and mortality worldwide: IARC CancerBase No. 10. Lyon, France: International Agency for Research on Cancer, 2010.

5. El-Serag HB. Hepatocellular carcinoma. N Engl J Med 2011;365:1118-27.

6. El-Serag HB. Epidemiology of viral hepatitis and hepatocellular carcinoma. Gastroenterology 2012;142:1264-73.e1.

7. Ni Y-H, Chang M-H, Wu J-F, et al. Minimization of hepatitis B infection by a 25 -year universal vaccination program. $J$ Hepatol 2012;57:730-5.

8. Ott JJ, Stevens GA, Groeger J, et al. Global epidemiology of hepatitis B virus infection: new estimates of age-specific HBsAg seroprevalence and endemicity. Vaccine 2012;30:2212-19.
9. Ly KN, Jian X, Klevens RM, et al. The increasing burden of mortality from viral hepatitis in the United States between 1999 and 2007. Ann Intern Med 2012;156:271-8.

10. Fattovich G, Bortolotti F, Donato F. Natural history of chronic hepatitis $B$ : special emphasis on disease progression and prognostic factors. J Hepatol 2008;48:335-52.

11. Chen CJ, Yang HI, lloeje UH, et al. Hepatitis B virus DNA levels and outcomes in chronic hepatitis B. Hepatology 2009;49(S5):S72-84.

12. Liaw YF. Hepatitis B virus replication and liver disease progression: the impact of antiviral therapy. Antivir Ther 2006;11:669-79.

13. Lok AS. Does antiviral therapy for hepatitis $B$ and $C$ prevent hepatocellular carcinoma? J Gastroenterol Hepatol 2011;26:221-7.

14. EASL. EASL Clinical Practice Guidelines: management of chronic hepatitis B virus infection. $J$ Hepatol 2012:57:167-85.

15. Shamliyan TA, MacDonald R, Shaukat A, et al. Antiviral therapy for adults with chronic hepatitis $B$ : a systematic review for a National Institutes of Health Consensus Development Conference. Ann Intern Med 2009;150:111-24.

16. Yang YF, Zhao W, Zhong YD, et al. Interferon therapy in chronic hepatitis $B$ reduces progression to cirrhosis and hepatocellular carcinoma: a meta-analysis. J Viral Hepat 2009;16:265-71.

17. Sung JJY, Tsoi KKF, Wong VWS, et al. Meta-analysis: treatment of hepatitis $B$ infection reduces risk of hepatocellular carcinoma. Aliment Pharmacol Ther 2008;28:1067-77.

18. Shen YC, Hsu C, Cheng CC, et al. A critical evaluation of the preventive effect of antiviral therapy on the development of hepatocellular carcinoma in patients with chronic hepatitis $\mathrm{C}$ or $\mathrm{B}$ : a novel approach by using meta-regression. Oncology 2012;82:275-89.

19. Papatheodoridis GV, Lampertico P, Manolakopoulos S, et al. Incidence of hepatocellular carcinoma in chronic hepatitis $B$ patients receiving nucleos(t)ide therapy: a systematic review. J Hepatol 2010;53:348-56.

20. Miyake Y, Kobashi H, Yamamoto K. Meta-analysis: the effect of interferon on development of hepatocellular carcinoma in patients with chronic hepatitis B virus infection. J Gastroenterol 2009;44:470-5.

21. Zhang C-H, Xu G-L, Jia W-D, et al. Effects of interferon treatment on development and progression of hepatocellular carcinoma in patients with chronic virus infection: a meta-analysis of randomized controlled trials. Int J Cancer 2011;129:1254-64.

22. Singal AK, Fontana RJ. Meta-analysis: oral anti-viral agents in adults with decompensated hepatitis B virus cirrhosis. Aliment Pharmacol Ther 2012;35:674-89.

23. Cammà $\mathrm{C}$, Giunta $\mathrm{M}$, Andreone $\mathrm{P}$, et al. Interferon and prevention of hepatocellular carcinoma in viral cirrhosis: an evidence-based approach. J Hepatol 2001;34:593-602.

24. Baffis V, Shrier I. Use of interferon for prevention of hepatocellular carcinoma in cirrhotic patients with hepatitis $B$ or hepatitis $C$ virus infection. Ann Intern Med 1999;131:696-701.

25. Wong GLH, Yiu KKL, Wong VWS, et al. Meta-analysis: reduction in hepatic events following interferon-alfa therapy of chronic hepatitis $B$ Aliment Pharmacol Ther 2010;32:1059-68.

26. Manolakopoulos S, Karatapanis S, Elefsiniotis J, et al. Clinical course of lamivudine monotherapy in patients with decompensated cirrhosis due to $\mathrm{HBeAg}$ negative chronic HBV infection. Am J Gastroenterol 2004;99:57-63.

27. Liaw Y-F, Sung JJY, Chow WC, et al. Lamivudine for patients with chronic hepatitis B and advanced liver disease. N Engl J Med 2004;351:1521-31.

28. Krogsgaard K. The long-term effect of treatment with interferon-alpha $2 a$ in chronic hepatitis B. The Long-Term Follow-up Investigator Group. The European Study Group on Viral Hepatitis (EUROHEP). Executive Team on Anti-Viral Treatment. J Viral Hepat 1998;5:389-97.

29. Higgins JP, Green S.eds Cochrane Handbook for Systematic Reviews of Interventions. Version 5.1.0 [updated March 2011]: the Cochrane Collaboration, Cochrane Handbook for Systematic Reviews of Interventions. Version 5.1.0 [updated March 2011], 2011.

30. Stroup DF, Berlin JA, Morton SC, et al. Meta-analysis of observational studies in epidemiology: a proposal for reporting. Meta-analysis Of Observational Studies in Epidemiology (MOOSE) group. JAMA 2000;283:2008-12.

31. EASL, EORTC. EASL-EORTC clinical practice guidelines: management of hepatocellular carcinoma. J Hepatol 2012;56:908-43.

32. Colli A, Fraquelli M, Casazza G, et al. Accuracy of ultrasonography, spiral CT, magnetic resonance, and alpha-fetoprotein in diagnosing hepatocellular carcinoma: a systematic review. Am J Gastroenterol 2006;101:513-23.

33. Higgins J, Altman D, Sterne J.eds Chapter 8: assessing risk of bias in included studies: the Cochrane Collaboration, Cochrane Handbook for Systematic Reviews of Interventions. Version 5.1.0 [updated March 2011]. 2011.

34. Wetterslev J, Thorlund $\mathrm{K}$, Brok J, et al. Trial sequential analysis may establish when firm evidence is reached in cumulative meta-analysis. J Clin Epidemiol 2008;61:64-75. 
35. Anderson MG, Harrison TJ, Alexander G, et al. Randomised controlled trial of lymphoblastoid interferon for chronic active hepatitis B. Gut 1987;28:619-22.

36. Benvegnù L, Chemello L, Noventa $\mathrm{F}$, et al. Retrospective analysis of the effect of interferon therapy on the clinical outcome of patients with viral cirrhosis. Cancer 1998;83:901-9.

37. Bolukbas C, Bolukbas FF, Kendir T, et al. The effectiveness of lamivudine treatment in cirrhotic patients with HBV precore mutations: a prospective, open-label study. Dig Dis $\mathrm{Sci}$ 2006;51:1196-202

38. Brunetto MR, Oliveri F, Coco B, et al. Outcome of anti-HBe positive chronic hepatitis $B$ in alpha-interferon treated and untreated patients: a long term cohort study. J Hepatol 2002;36:263-70.

39. Chan $\mathrm{HL}$, Wang $\mathrm{H}$, Niu J, et al. Two-year lamivudine treatment for hepatitis $\mathrm{B}$ e antigen-negative chronic hepatitis B: a double-blind, placebo-controlled trial. Antivir Ther 2007;12:345-53.

40. Chan SL, Mo FKF, Wong VWS, et al. Use of antiviral therapy in surveillance: impact on outcome of hepatitis B-related hepatocellular carcinoma. Liver Int 2012;32:271-8.

41. Das K, Das K, Datta S, et al. Course of disease and survival after onset of decompensation in hepatitis B virus-related cirrhosis. Liver Int 2010;30:1033-42.

42. Di Marco V, lacono OL, Cammà $\mathrm{C}$, et al. The long-term course of chronic hepatitis B. Hepatology 1999;30:257-64.

43. Farci $\mathrm{P}$, Roskams $\mathrm{T}$, Chessa $\mathrm{L}$, et al. Long-term benefit of interferon $\alpha$ therapy of chronic hepatitis $D$ : regression of advanced hepatic fibrosis. Gastroenterology 2004;126:1740-9.

44. Fattovich G, Giustina G, Realdi G, et al. Long-term outcome of hepatitis $B$ e antigen-positive patients with compensated cirrhosis treated with interferon alfa. Hepatology 1997;26:1338-42.

45. IIHCSG. Effect of interferon- $\alpha$ on progression of cirrhosis to hepatocellular carcinoma: a retrospective cohort study. Lancet 1998;351:1535-9.

46. Ikeda K, Saitoh S, Suzuki Y, et al. Interferon decreases hepatocellular carcinogenesis in patients with cirrhosis caused by the hepatitis B virus. Cancer 1998;82:827-35.

47. Lin CC, Wu JC, Chang TT, et al. Long-term evaluation of recombinant interferon $\alpha 2 b$ in the treatment of patients with hepatitis $\mathrm{B}$ e antigen-negative chronic hepatitis B in Taiwan. J Viral Hepat 2001;8:438-46.

48. Lin SM, Yu ML, Lee CM, et al. Interferon therapy in HBeAg positive chronic hepatitis reduces progression to cirrhosis and hepatocellular carcinoma. J Hepatol 2007;46:45-52.

49. Ma H, Wei L, Guo F, et al. Clinical features and survival in Chinese patients with hepatitis $B$ e antigen-negative hepatitis $B$ virus-related cirrhosis. J Gastroenterol Hepatol 2008;23(8pt 1): 1250-8.

50. Mahmood S, Niiyama G, Kamei A, et al. Influence of viral load and genotype in the progression of Hepatitis B-associated liver cirrhosis to hepatocellular carcinoma. Liver Int 2005;25:220-5.

51. Matsumoto A, Tanaka E, Rokuhara A, et al. Efficacy of lamivudine for preventing hepatocellular carcinoma in chronic hepatitis $B$ : a multicenter retrospective study of 2795 patients. Hepatol Res 2005;32:173-84.

52. Mazzella G, Accogli E, Sottili S, et al. Alpha interferon treatment may prevent hepatocellular carcinoma in HCV-related liver cirrhosis. $J$ Hepatol 1996;24:141-7.

53. Mazzella G, Saracco G, Festi D, et al. Long-term results with interferon therapy in chronic type $B$ hepatitis: a prospective randomized trial. Am J Gastroenterol 1999:94:2246-50.
54. Niederau C, Heintges T, Lange S, et al. Long-term follow-up of $\mathrm{HBeAg}$-positive patients treated with interferon alfa for chronic hepatitis B. N Engl J Med 1996;334:1422-7.

55. Papatheodoridis GV, Manesis E, Hadziyannis SJ. The long-term outcome of interferon-alpha treated and untreated patients with $\mathrm{HBeAg-negative} \mathrm{chronic} \mathrm{hepatitis} \mathrm{B.} \mathrm{J} \mathrm{Hepatol} \mathrm{2001;34:306-13.}$

56. Robson SC, Brice E, Van Rensburg C, et al. Safety and efficacy of interferon alpha-2b following prednisone withdrawal in the treatment of chronic viral hepatitis B. A case-controlled, randomised study. $S$ Afr Med J 1992;82:317-20.

57. Romeo R, Del Ninno E, Rumi M, et al. A 28-year study of the course of hepatitis $\Delta$ infection: a risk factor for cirrhosis and hepatocellular carcinoma. Gastroenterology 2009;136:1629-38.

58. Tangkijvanich $\mathrm{P}$, Thong-ngam $\mathrm{D}$, Mahachai $\mathrm{V}$, et al. Long-term effect of interferon therapy on incidence of cirrhosis and hepatocellular carcinoma in Thai patients with chronic hepatitis B. Southeast Asian $J$ Trop Med Public Health 2001;32:452-8.

59. Tong MJ, Blatt LM, Tyson KB, et al. Death from liver disease and development of hepatocellular carcinoma in patients with chronic hepatitis B virus infection: a prospective study. Gastroenterol Hepatol 2006;2:41-7.

60. Tong MJ, Hsien C, Song JJ, et al. Factors associated with progression to hepatocellular carcinoma and to death from liver complications in patients with HBsAg-positive cirrhosis. Dig Dis Sci 2009;54:1337-46.

61. Truong BX, Seo Y, Kato M, et al. Long-term follow-up of Japanese patients with chronic hepatitis B treated with interferon-alpha. Int $J$ Mol Med 2005;16:279-84.

62. Waked I, Amin M, Abd el Fattah S, et al. Experience with interferon in chronic hepatitis B in Egypt. J Chemother 1990;2:310-18.

63. Wong VW-S, Chan SL, Mo F, et al. Clinical scoring system to predic hepatocellular carcinoma in chronic hepatitis B carriers. J Clin Oncol 2010;28:1660-5.

64. Yuen M-F, Hui C-K, Cheng C-C, et al. Long-term follow-up of interferon alfa treatment in Chinese patients with chronic hepatitis $B$ infection: the effect on hepatitis $B$ e antigen seroconversion and the development of cirrhosis-related complications. Hepatology 2001;34:139-45.

65. Yuen M-F, Wong DK-H, Sablon E, et al. HBsAg seroclearance in chronic hepatitis $B$ in the Chinese: virological, histological, and clinical aspects. Hepatology 2004;39:1694-701.

66. Yuen MF, Seto WK, Chow DH, et al. Long-term lamivudine therapy reduces the risk of long-term complications of chronic hepatitis $B$ infection even in patients without advanced disease. Antivir Ther 2007;12:1295-303.

67. Zampino R, Marrone A, Merola A, et al. Long-term outcome of hepatitis $B$ and hepatitis $C$ virus co-infection and single HBV infection acquired in youth. J Med Virol 2009;81:2012-20.

68. Psaty BM, Weiss NS, Furberg CD, et al. Surrogate end points, health outcomes, and the drug-approval process for the treatment of risk factors for cardiovascular disease. JAMA 1999;282:786-90.

69. Odgaard-Jensen J, Vist GE, Timmer A, et al. Randomisation to protect against selection bias in healthcare trials. Cochrane Database Syst Rev 2011;(4):MR000012.

70. Kimer N, Dahl EK, Gluud LL, et al. Antiviral therapy for prevention of hepatocellular carcinoma in chronic hepatitis $\mathrm{C}$ : systematic review and meta-analysis of randomised controlled trials. BMJ Open 2012;2: e001313 doi:10.1136/bmjopen-2012-001313.

71. Savovic J, Jones HE, Altman DG, et al. Influence of reported study design characteristics on intervention effect estimates from randomized, controlled trials. Ann Intern Med 2012;157:429-38. 